\title{
PALMPRINT VERIFICATION USING INVARIANT MOMENTS BASED ON WAVELET TRANSFORM
}

\author{
Inass SH. Hussein and Md Jan Nordin \\ Faculty of Information Science and Technology, Universiti Kebangsaan Malaysia, UKM, Bangi, Malaysia
}

Received 2014-01-16; Revised 2014-02-27; Accepted 2014-03-07

\begin{abstract}
Data security is one of the important issues among computer users. Data security can prevent fraudulent users from accessing an individual's personal data. The biometrics recognition as one of the most important parts in the security of the data and the application of computer vision. The biometrics is the authentication method used in a wide variety of applications such as e-banking, e-commerce, e-government and many others. A biometric system is one which requires the recognition of a pattern, whereby it enables the differentiation of features from one individual to another. Biometric technologies, thus may be defined as the automated methods of identifying, or authenticating, the identity of a living person based on physiological or behavioral traits. This study emphasizes palmprint recognition, which provides a wide deployment range of authentication methods. The palmprint contains principal lines, wrinkles, fine lines, ridges and surface area; thus the palmprint of person differs from one to another. Previous researchers have difficulty extracting the features of a palm print, because of the effects of rotation, translation and scaling changes and the accuracy rate of verification performance needs to be improved. The aim of this study is to extract shape features using an invariant moments algorithm based on wavelet transform and identify the person's verification. This model has shown a promising results without the effects of rotation, translation and scaling of objects, because it is associated with the use of a good description of shape features. This system has been tested using databases from the Indian Institute of Technology, Kanpur (IITK), by using the False Rejection Rate (FRR) and False Acceptance Rate (FAR), we may calculate the accuracy rate of verification. The experiment shows a $97.99 \%$ accuracy rate of verification.
\end{abstract}

Keywords: Biometric System, Verification, Invariant Moments, Roi, 2D Wavelet Ttransform

\section{INTRODUCTION}

The developments that have occurred in information technology have assisted human beings in performing tasks which were previously difficult to carry out, or manually carried out. Indirectly, these developments have helped mankind by providing faster, more efficient and productive performance of tasks. There are a number of problems which make mankind use information technology to get more accurate results. The issue of security is one problem encountered by all countries in the world (Ribaric and Fratric, 2005). The applications of image processing may be used in many fields such as security, pattern recognition, compression and computer vision. Various ways and techniques have been presented to surmount security problems. One such technique is the use of biometrics (Zhang et al., 2003; Duta, 2009). Biometrics refers to the use of physiological or biological features to recognize the identity of an individual. These characteristics uniquely exist in each individual and keep unchanged during a person's lifetime. These traits make biometrics a promising solution to society as a whole. A knowledge based security is one technique of security in which the access to the secured zone may be made by the use of ID numbers or passwords. However, such information may easily be accessed and breached by intruders. The problem occurs in the case of monetary transactions and Corresponding Author: Inass SH. Hussein, Faculty of Information Science and Technology, Universiti Kebangsaan Malaysia, UKM, Bangi, Malaysia 
highly limited in the information area. Thus, to solve the above mentioned issue, biometric features are used. A biometric system is basically a pattern recognition system which identifies a person by determining the authenticity of a specific physiological or behavioral features possessed by the user. Biometric technologies, thus may be defined as the automated methods of identifying or authenticating the identity of a living person based on physiological or behavioral traits.

Biometrics based personal identification is as an effective method for automatically recognizing, a persons identity with high confidence (Gayathri and Ramamoorthy, 2012a). Biometric recognition systems are able to identify the person depending on the feature vector of any one of the biometrics that the person possesses (Delac and Gregic, 2004). These systems are used for authorizing a person to reach the secured zone. Thus, they provide a more secure and convenient identification compared with alternative methods of identification. The systems use computer-based security in various commercial applications, whether civil and criminal applications. Each person has to determine the identity of the starting of the license in order for drivers to gain entry into the country of the passport. The system also uses the physical properties of the individual in the biometric system, such as fingerprints, hand geometry, face, voice or iris. They are more reliable and secure because they provide access to authorized users in the physical presence (Jain et al., 2006). This study used a palmprint recognition to identify a person's authentication. Palmprint recognition is an efficient biometric solution for authentication system. The existence of several hand-based authentication commercial systems indicates the effectiveness of this type of biometric (Gayathri and Ramamoorthy, 2012b).

The authentication may be defined as giving authorized persons the suitable authorities and access at the proper time (Woodward et al., 2003). The classification of personal authentication problems may be categorized into two classes, which are verification and identification.

The verification problem is the process of determining whether a certain person is the claimed person, or a person pretending to be the original. The verification problem may be described as one-to-one. The matching of patterns to be identified may be done by matching with only one template that is claimed person (Jain et al., 2004).

The palmprint contains principal lines, wrinkles, fine lines, ridges and surface area; thus the palmprint of a person differs from one to another, as shown in Fig. 1.

\section{MATERIALS AND METHODS}

The basic objective of this study is to build a system for recognizing palmprints with high accuracy and performance. In this study, a method is proposed to overcome limitations of previous studies and improve accuracy of palmprint recognition systems. The methodology of the research is a set of processes which has been regularly organized for generating information. The methodology has indirectly benefited the researcher in achieving the objectives and results of the study.

The aim of this study is to propose an invariant moment's algorithm based on wavelet transform to improve the accuracy rate and time performance and extract highly distinctive features from the palmprint without the effects of rotation, translation and scaling changes from one individual to another. The algorithm used to derive multi invariant moments for all input images palmprint. The method used in the experiment has two parts. The first part involves extracting shape features based on wavelet transform; the second part is verification based on promising matches algorithm. The following section contains details of the proposed method for palmprint recognition. Each phase in the methodology is explained below.

\subsection{Hand Image Acquisition}

This study uses one set of data for testing: From the IITK dataset. The acquisition of the differences of the object is a challenge in the object is set correctly for this category. The first step was the extraction of palmprint from hands.

The IITK database consists of 600 left and right hand images that were obtained from 50 users through the low-cost flatbed scanner. All images are $800 \times 600$ pixels in size. The flatbed scanner has 256 levels of gray. The device is peg-free, so the user is free to rotate his/her hand by $\pm 35^{\circ}$ symmetrically to the working surface of the scanner. Figure 2 shows a sample of a user's hand. These hands have different rotation degrees and sizes.

\subsection{The Proposed Step for Pre-Processing}

The preprocessing phase is considered as the most important phase in processing digital images because it is a critical phase of digital image processing, since it helps to remove noise. The pre-processing stage enhances the contrast of the acquired image, which means that undesirable information not related to the task is withheld (González and Woods, 1993). This stage calls for transforming images by altering their tone to eliminate or diminish background noise, enhance edges and to highlight the feature of interest. The preprocessing phase enhances resulting images for the next step of palmprint recognition. 


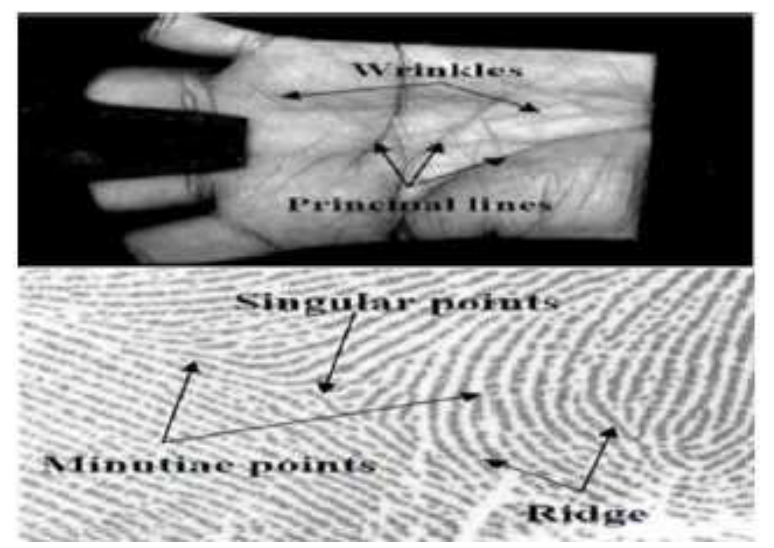

Fig. 1. Shape and details of palmprint
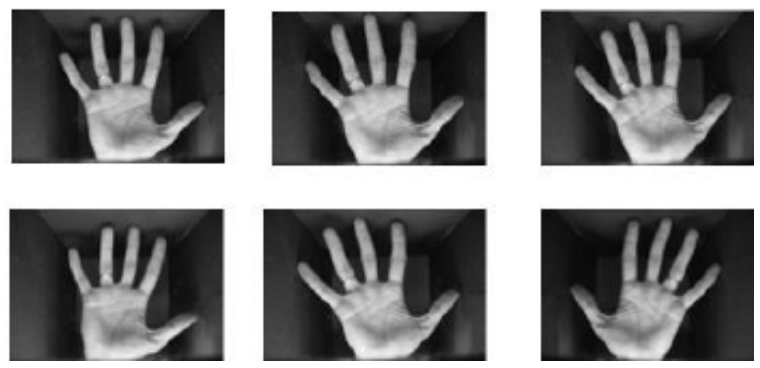

Fig. 2. The hands of one user from the IITK database

\subsection{Binarized Hand Image from the Original Image}

At this stage, global threshold is applied to extract the image from the rear hand. This step is processed to be accomplished before extracting the region of the palmprint of the left and right hands. Unification must be analyzed backlight while capturing images and contrasting colors of the background images to control the contrasting colors of the images, as shown in Fig. 3.

\subsection{Extraction of the Palmprint Region from the Whole Hand (ROI)}

At this stage, the contour tracing algorithm is used in the image of the hand binarized. Figure 4 shows the contour of the hand's image.

To find the start point of pixels, the object (hand image) pixel border may be chosen as a starting point, provided the left adjacent pixels is not an object. Next, scan each column of pixels from the bottom going up, starting from the column on the far left and then moving to the right. This process continues until an object pixel is encountered (Pavlidis, 1982). Than finding the best pixel following, let $\mathrm{P}$ be the current pixel border and $\mathrm{P} 1$, P2 and P3 be adjacent pixels:
- If P1 is the object's pixel, then this will be the next border pixel

- Otherwise, P2 is the object's pixel it will be the border pixel

- If P1 and P2 are both background pixels, then, P3 will be the next border pixel

This procedure continues until it finds an object's pixel. The pixel is considered an isolated pixel in the case there are no neighboring pixels found for all possible neighborhood set shown in Fig. 5.

The region of interest on the palmprint is the square area with two of its corners and placed at the middle point of the line segments $\mathrm{C} 1-\mathrm{V} 1$ and $\mathrm{V} 2-\mathrm{C} 2$ respectively. The line segments $\mathrm{C} 1-\mathrm{V} 1$ and $\mathrm{V} 2-\mathrm{C} 2$ are at an angle of $45^{\circ}$ and $60^{\circ}$, respectively, to join the line V1 and V2. Figure 6 shows the points and the relevant areas of interest on the palm print.

\subsection{Feature Extraction}

In this proposed method, we will extract the shape feature by using an invariant moments algorithm based on wavelet transform. First, we applied 2D wavelet transform (2D filter bank) into (ROI) of the palmprint, that will decompose the (ROI) into four bands, low-low band (LL), Low-High band (LH), high-low band (HL) and HighHigh (HH) (Abidin et al., 2013) as shown in Fig. 7.

$\mathrm{Next}$, we applied the $\mathrm{Hu}$ moments algorithm into the decomposed image, to derive seven moments from each band. Hu et al. (2007) defines seven of these shape descriptor values computed from central moments through order three that are independent to object translation, scale and rotation. Translation invariance is achieved by computing moments that are normalized with respect to the center of gravity, so that the center of mass of the distribution is at the origin (central moments). The size of invariant moments is derived from algebraic invariants, but these may be shown to be the result of simple size normalization. From the second and third order values of the normalized central moments, a set of seven invariant moments may be computed which are independent of rotation. Traditionally, moments invariant are computed based on the information provided by both the shape boundary and its interior region. The moments used to construct the moment invariant are defined in the continuous, but for practical implementation they are calculated in the discrete phase. Given a function $\mathrm{f}(\mathrm{x}, \mathrm{y})$, we will assume that $f(x, y)$ is a non-negative function with finite values on a bounded image plane. This is appropriate for most image applications. These regular moments are defined by Equation 1:

$\mathrm{m}_{\mathrm{pq}}=\int_{-\infty}^{\infty} \int_{-\infty}^{\infty} \mathrm{x}^{\mathrm{p}} \mathrm{y}^{\mathrm{q}} \mathrm{f}(\mathrm{x}, \mathrm{y}) \mathrm{dxdy}$ 


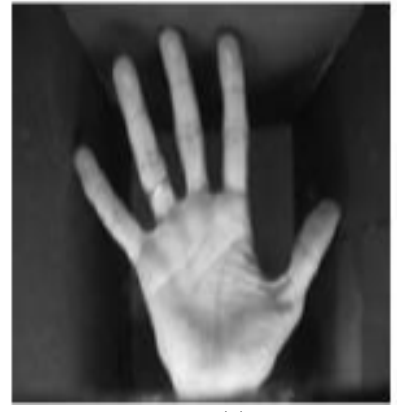

(a)

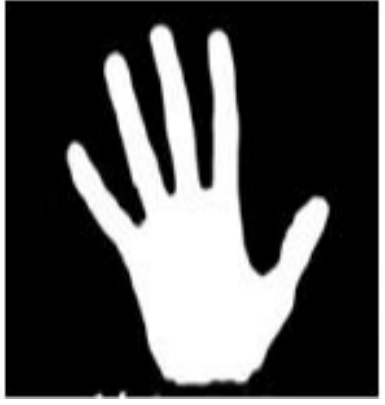

(b)

Fig. 3. (a) Original image, (b) binarized hand image
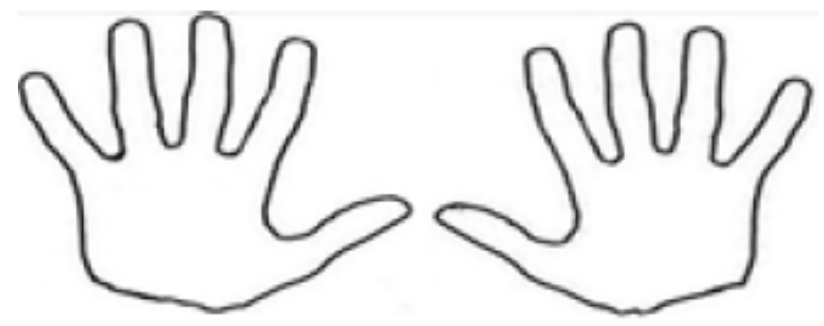

Fig. 4. Hand contour

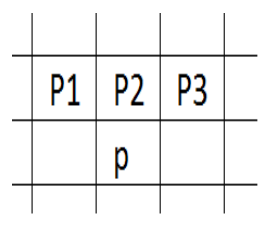

(a)

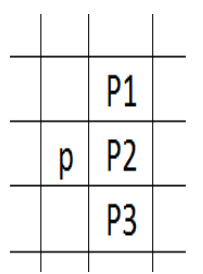

(b)

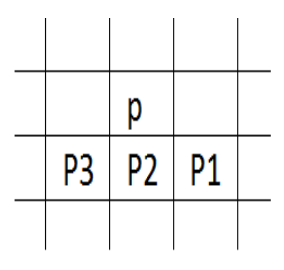

(c)

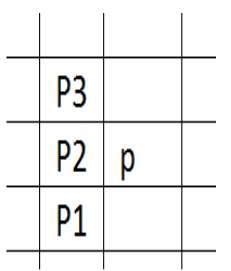

(d)

Fig. 5. Possible neighborhood pixel
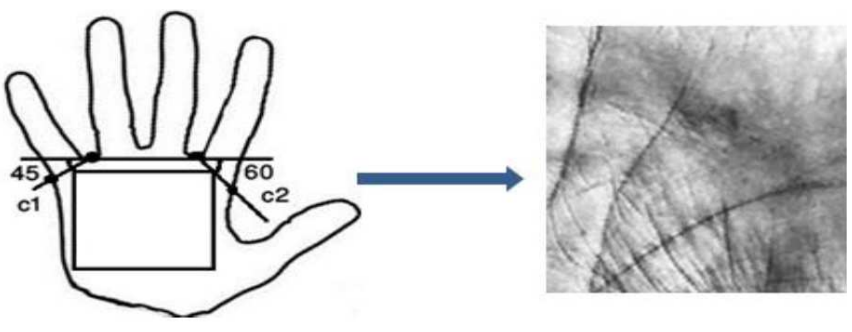

Fig. 6. Related points and regions of interest of palmprint

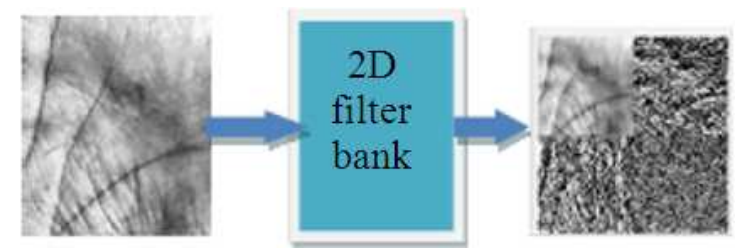

Fig. 7. ROI image and decomposes image
For $\mathrm{p}, \mathrm{q}=0,1,2,3$.

It is assumed that the function $\mathrm{f}(\mathrm{x}, \mathrm{y})$ is $2-\mathrm{D}$ continuous and uniqueness theorem may be proved.

A uniqueness theorem (Papoulis, 1991) states that iff $(x, y)$ is piecewise continuous and has non zero values only in a finite part of the $\mathrm{x}$, $\mathrm{y}$ plane, moments of all orders exist and the moment sequence (mpq) is 
uniquely determined by $\mathrm{f}(\mathrm{x}, \mathrm{y})$. Conversely, (mpq) uniquely determines if ( $\mathrm{x}, \mathrm{y})$ Equation 3 and 4:

$\mu_{\mathrm{pq}}=\int_{-\infty}^{\infty} \int_{-\infty}^{\infty}(\mathrm{x}-\overline{\mathrm{x}})^{\mathrm{p}}(\mathrm{y}-\overline{\mathrm{y}})^{\mathrm{q}} \mathrm{f}(\mathrm{x}, \mathrm{y}) \mathrm{dxdy}$

$\bar{x}=\frac{\iint x f(x, y) d x d y}{\iint f(x, y) d x d y}=\frac{M_{10}}{M_{00}}$

$\bar{y}=\frac{\iint y f(x, y) d x d y}{\iint f(x, y) d x d y}=\frac{M_{01}}{M_{00}}$

The total mass of the function is given from the moment $\mathrm{m} 00$. When the function has been normalized so that it has unit mass it is sometimes called a probability function. For a digital image (i.e., discrete function), Equation 2 will be Equation 5:

$\mu_{\mathrm{pq}}=\sum_{\mathrm{x}=1}^{\mathrm{n}} \sum_{\mathrm{y}=1}^{\mathrm{n}}(\mathrm{x}-\overline{\mathrm{x}})^{\mathrm{p}}(\mathrm{y}-\overline{\mathrm{y}})^{\mathrm{q}} \mathrm{f}(\mathrm{x}, \mathrm{y})$

The central moment is obtained by shifting the origin to the centroid of the function. To order up to 3 are Equation 6 to 10:

$$
\begin{aligned}
& \mu_{00}=M_{00} \\
& \mu_{11}=M_{11}-\bar{Y} M_{10} \\
& \mu_{10}=0 \\
& \mu_{30}=M_{30}-3 \bar{X} M_{20}+2 \bar{X}^{2} M_{10} \\
& \mu_{01}=0 \\
& \mu_{12}=M_{12}-2 \bar{Y} M_{11}-\bar{X} M_{02}+2 \bar{Y}^{2} M_{01} \\
& \mu_{20}=M_{20}-\bar{X} M_{10} \\
& \mu_{21}=M_{21}-2 \bar{X} M_{11}-\bar{Y} M_{11}-\bar{Y} M_{20}+2 \bar{X}^{2} M_{01} \\
& \mu_{02}=M_{02}-\bar{Y} M_{01} \\
& \mu_{30}=M_{03}-3 \bar{Y} M_{02}+2 \bar{Y}^{2} M_{01}
\end{aligned}
$$

This set of central moment equations may be normalized under scaling by multiplying by each of the above equations by the factor $\frac{1}{\mu_{00}^{\gamma}}$.
Where:

$$
\gamma=\frac{\mathrm{p}+\mathrm{q}}{2}+1
$$

For $\mathrm{p}+\mathrm{q}=2,3,4, \ldots \ldots$

So the normalized central moment, denoted by Equation 11:

$$
\eta_{\mathrm{pq}}=\frac{\mu_{\mathrm{pq}}}{\mu_{00}^{\gamma}}
$$

From the second and third moment, a set of seven invariant moments may be derived. They are given by:

$$
\begin{aligned}
& \Phi_{1}=\eta_{20}+\eta_{02} \\
& \Phi_{2}=\left(\eta_{20}-\eta_{02}\right)+4 \eta_{11}^{2} \\
& \Phi_{3}=\left(\eta_{30}-3 \eta_{12}\right)^{2}+\left(3 \eta_{21}-\eta_{03}\right)^{2} \\
& \Phi_{4}=\left(\eta_{30}+\eta_{12}\right)^{2}+\left(\eta_{21}+\eta_{03}\right) \\
& \Phi_{5}=\left(\eta_{30}-3 \eta_{12}\right) \\
& \left(\eta_{30}+3 \eta_{12}\right)^{2}\left[\left(\left(\eta_{30}+\eta_{12}\right)^{2}\right.\right. \\
& -3\left(\left(\eta_{21}+\eta_{30}\right)^{2}\right]+ \\
& \left({ }^{3} \eta_{21}-\eta_{03}\right)\left(\eta_{21}+\eta_{03}\right) \\
& \left.\Phi_{6}=\left(\eta_{20}-\eta_{02}\right)\left[\left(\eta_{30}+\eta_{12}\right)^{2}\right]-\left(\eta_{21}+\eta_{03}\right)^{2}\right] \\
& +4 \eta_{11}\left(\eta_{30}+\eta_{12}\right)\left(\eta_{21}+\eta_{03}\right) \\
& \Phi_{7}=\left(3 \eta_{21}-\eta_{03}\right)\left(\eta_{30}+\eta_{12}\right)\left[\left(\eta_{30}+\eta_{12}\right)^{2}\right. \\
& \left.\left.-3\left(\eta_{21}+\eta_{03}\right)^{2}\right]+() 3 \eta_{12}-\eta_{30}\right)\left(\eta_{21}+\eta_{03}\right) \\
& {\left[3\left(\eta_{30}+\eta_{12}\right)^{2}-\left(\eta_{21}+\eta_{03}\right)^{2}\right]}
\end{aligned}
$$

\subsection{Verification}

Verification is the process of matching one image against one specific image which is carrying the same Id. If we suppose the query image is an $X$ and the image in the database is $\mathrm{X}$. We must compare $\mathrm{X}$ with $X$. In the design and implementation of an efficient palmprint system for the purpose of verification, the matching stage is considered the most important part of palmprint recognition, at the matching stage, several issues, such as the technique used in feature extraction, the complexity of images, the time of processing must be considered. The important points of interest in the verification of the palmprint are the features of an image, the change in the scale and rotation of the image's features. Here, we used a developed algorithm to compare two numbers 
(moments values of a query image with moments values of the image in the database). To test our model, we calculate both the False Rejection Rate (FRR) and the False Acceptance Rate (FAR). Finally, we determined the accuracy rate of verification based on two factors above, as: (Accuracy $=100$ $((\mathrm{FRR}+\mathrm{FAR}) / 2))$.

\section{RESULTS}

The proposed method was tested and evaluated by a phase having 50 persons. By finding FRR and FAR, we could consequently find the accuracy rate of the verification phase. After applying wavelet transform on palmprint images and decomposing these images into four bands, the invariant moments algorithm was applied for deriving seven moments from each band, with a total of 28 moments to the whole image. Table 1 shows an example of seven moments of four bands for one user from the IITK database.

After that, we applied the proposed algorithm to reduce the values of moments to seven moments and to spread the error to the whole image (by combining first moments for each band and dividing the total value by four and so on for all other bands). Table 2 shows the final seven moments which were derived from Table 1.

Finally, to perform verification in our proposed method and calculate the accuracy rate of this phase, the matching is performed between one query image and a specific image in the database carrying the same Id. Then, both FAR and FRR were calculated. Where to calculate the FRR factor, samples of five sets were taken, the size of each set 15 palmprint template, these samples belong to the same stored database and carrying the same Id. In order to calculate the FAR, samples of five sets were taken, the size of each set 15 palmprint templates, these samples were not included in the database and some of these samples may be carrying same Id of the stored database. Table 3 shows the final results.

The results show that the highest accuracy is obtained when both FAR and FRR are equal to zero, this as in the set no. 1, 4 and 5. The rate of accuracy is $97.99 \%$. Figure 8 shows the graph of accuracy rate.

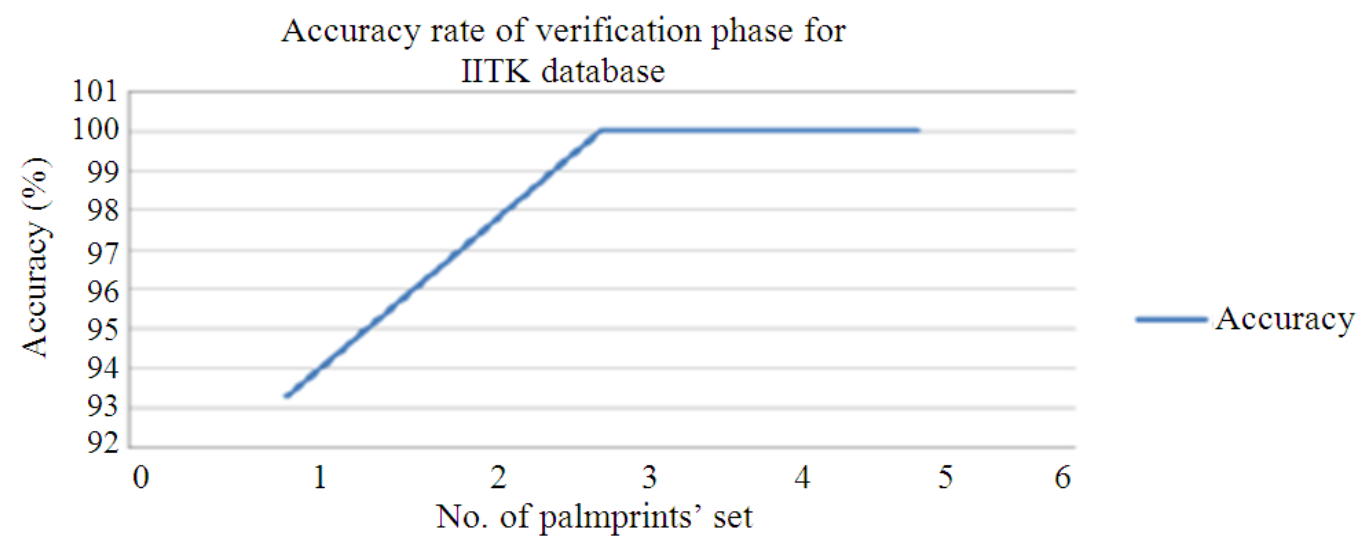

Fig. 8. Accuracy rate

Table 1. Values of the seven moments of four bands of one palmprint user from IITK the database

\begin{tabular}{lllll}
\hline Moments & LL & LH & HL & HH \\
\hline First & $1.058316619 \mathrm{E}-03$ & $3.595313987 \mathrm{E}-02$ & $3.081314579 \mathrm{E}-02$ & $6.989177985 \mathrm{E}-02$ \\
Second & $6.605290396 \mathrm{E}-09$ & $1.027599831 \mathrm{E}-06$ & $9.753945546 \mathrm{E}-06$ & $1.696854068 \mathrm{E}-05$ \\
Third & $2.950107454 \mathrm{E}-12$ & $4.320028904 \mathrm{E}-07$ & $3.460891563 \mathrm{E}-07$ & $5.109634664 \mathrm{E}-06$ \\
Fourth & $3.050136038 \mathrm{E}-12$ & $8.173558082 \mathrm{E}-07$ & $1.087733126 \mathrm{E}-06$ & $9.054234981 \mathrm{E}-06$ \\
Fifth & $-1.78670562 \mathrm{E}-20$ & $9.714754791 \mathrm{E}-11$ & $5.061546419 \mathrm{E}-11$ & $3.385753709 \mathrm{E}-09$ \\
Sixth & $-1.46878706 \mathrm{E}-17$ & $1.557931291 \mathrm{E}-10$ & $-1.37406396 \mathrm{E}-09$ & $8.165301536 \mathrm{E}-09$ \\
Seventh & $9.099224706 \mathrm{E}-24$ & $2.418751001 \mathrm{E}-14$ & $3.338217445 \mathrm{E}-13$ & $2.147370405 \mathrm{E}-11$ \\
\hline
\end{tabular}


Table 2. Final seven moments derived from Table 1

\begin{tabular}{ll}
\hline Moment & Value \\
\hline First moment & $3.442909549 \mathrm{E}-02$ \\
Second moment & $6.939172833 \mathrm{E}-06$ \\
Third moment & $1.471932414 \mathrm{E}-06$ \\
Forth moment & $2.739831742 \mathrm{E}-06$ \\
Fifth moment & $8.833791788 \mathrm{E}-10$ \\
Sixth moment & $1.736757682 \mathrm{E}-09$ \\
Seventh moment & $5.457928318 \mathrm{E}-12$ \\
\hline
\end{tabular}

Table 3. Final results

\begin{tabular}{lllc}
\hline No. set & FRR $(\%)$ & FAR $(\%)$ & Accuracy $(\%)$ \\
\hline 1 & 0.00 & 0.00 & 100.00 \\
2 & 6.66 & 6.66 & 93.33 \\
3 & 6.66 & 0.00 & 96.66 \\
4 & 0.00 & 0.00 & 100.00 \\
5 & 0.00 & 0.00 & 100.00 \\
Rate & 2.66 & 1.33 & 97.99 \\
\hline
\end{tabular}

Table 4. Comparison of verification accuracy of IITK database between our proposed method and some previous work

\begin{tabular}{lr}
\hline Method & IITK database (\%) \\
\hline Non weighted fusion (Kumar, 2009) & 92.32 \\
Weighted fusion (Kumar, 2009) & 92.74 \\
Proposed method & 97.99 \\
\hline
\end{tabular}

\section{DISCUSSION}

The main contribution of this work is a new method to extract shape features by using invariant moments algorithm based on wavelet transform. This method is promising, as it has obtained $97.99 \%$ accuracy. From this method, we can extract an very accurate shape feature when compared with previous methods. Generally, it exhibits very good results in the rotation, translation and scaling of a palmprint image, as shown in the experiments above. The results of these experiments clearly proved that the proposed model can get high accuracy verification. This model may be the foundation for future work on extracting shape features by using invariant moments algorithm based on wavelet transform Table 4.

\section{CONCLUSION}

This study was done based on methods considered important steps in processing and palmprint recognition. These steps include the major task of processing, feature extraction and matching, which were carried in a variety of ways and algorithms that are suitable for the structural condition of the image itself. The results of the experiment generally proved that the objective of using invariant moments based on wavelet transform is successfully implemented on palmprints. Moments extraction from the palmprint is found to be invariant to rotation, translation and scaling. Wavelet transform was applied to decompose the palmprint image that made invariant moments more effected to achieve its objective.

From the testing process we noticed that, the accuracy of the system depend on many factors such as: (i) the acquisition of images, (ii) resolution of images, (iii) size of the samples that used in testing and (v) the size of the database of the system.

Future work can involve combining Invariant Moments with some method such Zernike-Moments and Legendre Moments for improving verification accuracy.

\section{ACKNOWLEDGEMENT}

The researchers wish to thank Universiti Kebangasaan Malaysia (UKM) and Ministry of Higher Education Malaysia for supporting this work by research grant ERGS/1/2012/STG07/UKM/02/9.

\section{REFERENCES}

Abidin., Z., M. Manaf, A. Ahmad, Z. Ayop and S. Anawar et al., 2013. Online palmprint identification. Aus. J. Basic Applied Sci., 7: 46-50.

Delac, K. and M. Gregic, 2004. A survey of biometric recognition methods. Proceedings of the 46th International Symposium Electronics in Marine, Jun. 18-18, IEEE Xplore Press, Zadar, Croatia, pp: 184-193.

Duta, N., 2009. A survey of biometric technology based on hand shape. Patt. Recogn., 42: 2797-2806. DOI: 10.1016/j.patcog.2009.02.007

Gayathri, R. and P. Ramamoorthy, 2012a. Automatic personal identification using feature similarity index matching. Am. J. Applied Sci., 9: 678-685. DOI: 10.3844/ajassp.2012.678.685

Gayathri, R. and P. Ramamoorthy, 2012b. Automatic palmprint identification based on high order zernike moment. Am. J. Applied Sci., 9: 759-765. DOI: 10.3844/ajassp.2012.759.765

González, R.C. and R.E. Woods, 1993. Digital Image Processing. 1st Edn., Addison Wesley Reading, Massachusetts, pp: 716.

Hu, D., G. Feng and Z. Zhou, 2007. Two dimensional locality preserving projection (2dlpp) with its application to palm print recognition. Pattern Recognition, 40: 339-342. DOI: 10.1016/j.patcog.2006.06.022 
Jain, A.K., A. Ross and S. Pankanti, 2006. Biometrics. A tool for information security. IEEE. Trans. Inform. Forens. Security, 1: 125-143. DOI: 10.1109/TIFS.2006.873653

Jain, A.K., A. Ross and S. Prabhakar, 2004. An introduction to biometric recognition. IEEE. Trans. Circ. Syst. Video Technol., 14: 4-20. DOI: 10.1109/TCSVT.2003.818349

Kumar, K., 2009. An efficient occlusion invariant palmprint based verification system. MSc, Thesis, Indian Institute of Technology Kanpur.

Papoulis, A., 1991. Probability Random Variables and Stochastic Processes. 3rd Edn., Mcgraw-Hill, Incorporated, New York, ISBN-10: 0070484775, pp: 666.
Pavlidis, T., 1982. Algorithms for graphics and image processing, computer science press. Rockville, Maryland, 416. DOI: 10.1007/978-3-642-93208-3

Ribaric, S. and I. Fratric, 2005. A biometric identification system based on eigenpalm features. IEEE. Trans. Pattern Analysis Machine Int., 27: 1968-1709. DOI: 10.1109/TPAMI.2005.209

Woodward, J., H. Christopher, G. Julius and T. Aryn, 2003. Biometrics: A look at Facial Recognition. RAND Public Safety and Justice.

Zhang, D., W.K. Kong, J. You and M. Wong, 2003. Online palmprint identification. Trans. Patt. Anal. Machine Int., 25: 1041-1050. DOI: 10.1109/TPAMI.2003.1227981 